\title{
UNA APROXIMACIÓN ESTRUCTURALISTA A «EL CAPITAL» DE MARX A RAÍZ DEL PROBLEMA DE LA TRANSFORMACIÓN
}

\section{A STRUCTURALIST APPROACH TO MARX'S «CAPITAL» BASED ON THE TRANSFORMATION PROBLEM}

\author{
Josu Zabaleta IMAZ* \\ Universidad Autónoma de Madrid
}

Resumen: Se propone una lectura estructuralista de «El Capital» de Marx con la que enfrentarse a sus interpretaciones «fisicalistas» habituales. Bajo este nuevo enfoque, el controvertido papel del tercer tomo se convertirá en algo estructuralmente exigido por el primero. Para ello se mostrará el carácter isomorfo del mercado de mercancías y del mercado de capitales. La necesidad de contar con al menos dos materializaciones de la misma estructura para obtener su identificación dará lugar al concepto de «precio de producción».

PALABRAS CLAVE: Marx, estructura, isomorfismo, valor, precio, problema de la transformación.

ABSTRACT: A structuralist approach to Marx’s «Capital» is proposed as a way to confront the usual "physicalist» interpretations. From this new standpoint, the controversial role of the third volume will be transformed into something structurally required by the first volume. To this end, the isomorphic character of the market of goods and the markets of capitals will be shown. The need to have at least two materializations of the same structure available in order to be able to identify it gives rise to the concept of "price of production.»

KEYwORDS: Marx, structure, isomorphism, value, price, transformation problem.

* Dirección postal: Sorauerstr. 31, 10997, Berlín. E-mail: josu.zabaleta.imaz@googlemail.com. Este trabajo se ha realizado bajo los auspicios del Proyecto de Investigación: Pensar Europa: democracia y hegemonía en la era tecnológica. FFI 2009-10097. 
Se podría decir que prácticamente la totalidad ${ }^{1}$ de las intérpretes del «El Capital» $(D K)$ de Marx han considerado que el objeto de estudio de la obra, a saber, la mercancía presenta dos aspectos contrapuestos. Por una parte estaría el aspecto físico de la mercancía, el lado material de la misma. Dentro de este lado estarían incluidas las horas de trabajo físicamente medibles que han sido necesarias en la producción de la mercancía. Frente a este aspecto material se situaría el lado, por así decir, nominal de la mercancía. El primer aspecto expresaría el lado del valor de mercancía mientras que el aspecto nominal sería recogido por el precio de la mercancía. Desde esta lectura, sería perfectamente posible calcular el valor de una mercancía antes de que ésta pase por el mercado, es decir, antes de que haya sido vendida. El primer tomo del $D K$ sería la encargada de analizar el primer aspecto de la mercancía mientras que el segundo aspecto sería objeto de estudio del tercer tomo. El problema de expresar la relación entre el primer y el segundo aspecto de la mercancía, el denominado problema de la transformación, sería así el problema de pasar de los valores a los precios de las cercanías.

En el trabajo que ahora presentamos ofrecemos una interpretación del $D K$ que se distanciará tajantemente de esta lectura ya ortodoxa. Tal y como hemos adelantado en el título, la lectura que vamos a defender del $D K$ podría denominarse estructuralista. Y esto por varias razones. En primer lugar, debido a que disociaremos un plano material o empírico y un plano estructural o transfísico en la obra que es objeto de nuestro estudio. Veremos que esta división no sólo no coincide con los dos aspectos que la lectura tradicional detectaba en la mercancía sino que es incluso diametralmente opuesta a ella. Ello se debe a

\footnotetext{
${ }^{1}$ Cf. entre otros, E. Böhm-Bawerk: «Zum Abschluß des Marschen Systems», en: F. Eberle (ed.): Marxschen Theorie 1. Zur methodischen Bedeutung des 3. Bandes des ,Kapital, Frankfurt am Main, Suhrkamp, 1973, pp. 25-129, V. Bortkevi , Wertrechnung und Preisrechnung im marxschen System, Lollar/Gießen, Achenbach, 1976, L. R. Meek, "Some Notes on the "Transformation Problem»", The Economic Journal (Edinburgh), n 261, Marzo 1956, pp. 94-107, M. Morishima y G. Catephores, «Is There an "Historical Transformation Problem»?», The Economic Journal (Edinburgh), n 338, Junio 1975, pp. 309-328, P. Samuelson, "Understanding the Marxian Notion of Exploitation: A Summary of the So-Called Transformation Problem Between Marxian Values and Competitive Prices», Journal of Economic Literature (Nashville), $\mathrm{n}^{\circ}$ 2, Junio 1971, pp. 339-431, F. Seton "The "Transformation Problem»", The Review of Economic Studies (Oxford), n 3, Junio 1957, pp. 149-160 y P. M. Sweezy, The theory of capitalist development, Oxford, Oxford University Press, 1942.
} 
que el lado del valor, lado que para la lectura tradicional constituía el aspecto físico de la teoría de Marx, será, en nuestra interpretación, el elemento estructural de esta teoría. El lado empírico o positivo será proporcionado por los precios de las mercancías. En segundo lugar, la lectura que defendemos será estructuralista en el sentido de que la estructura económica que detectaremos lo será siempre de una u otra materia. Esto significa que no tiene sentido hablar de algo que es estructural como si de algo físico o físicamente medible se tratara. De este modo, veremos que no hay forma de detectar el valor si no es a través de su manifestación empírica o precio. En tercer lugar, veremos que habrá un elemento que, si bien se dará siempre en la experiencia, tendrá un origen irreductiblemente estructural. Este elemento será el plusvalor. Por último, en sintonía con la distinción entre el plano semántico y el plano fonético del análisis estructural del lenguaje, procederemos a identificar en el $D K$ de Marx una segunda materialización de la misma estructura. Esta nueva materialización, el mercado de capitales, permitirá detectar de una manera estrictamente relacional las identidades y diferencias que pueda haber en la primera materialización y viceversa.

\section{Valor de uso inmediato, Valor y Plusvalor}

El valor de la mercancía es aquello que «tiene» la mercancía en tanto que mercancía. El valor es una propiedad social de la mercancía. Esto significa que el valor no es algo físico, no es algo que exista por el hecho de que tal cosa tenga una Cantidad o una Cualidad determinadas, sino que el valor se manifiesta en la Relación que una mercancía tiene con otras mercancías. La objetividad propia del valor es la objetividad relacional. La relación que caracterizará a la sociedad moderna, relación en la que se manifiesta el valor de la mercancía es la relación de intercambio. En la forma de intercambio más inmediata se ponen en relación de igualdad cantidades concretas de dos mercancías concretas. Una unión de tal cantidad de tal mercancía es igualada a otra unión de tal cantidad de tal mercancía. Se trata de una relación en la que se mide algo por algo. En esta relación de intercambio, la mercancía medida se nos manifiesta bajo la forma relativa del valor «Relative Wertform». Por su parte, la mercancía que mide a la primera se manifiesta bajo lo que Marx denomina «Äquivalentform» o forma equivalente de valor. Esta mercancía singular (Einzelne) se intercambia por esta otra mercancía singular. 
Pero nada impide que esta misma mercancía se intercambie además con otras mercancías. En este caso la mercancía en cuestión será una entre otras muchas, es decir, la mercancía será una mercancía particular (Besondere). Bajo el supuesto de la competencia, es decir, suponiendo que los beneficios que son posibles realizar en la venta de una mercancía no derivan de una situación más o menos extrema de monopolio, o para decir lo mismo de otra forma, bajo el supuesto de que las ganancias son algo estructural y no anecdótico al sistema que intentamos describir, tendremos que la relación «ser intercambiable con» será lo que en lógica se denomina una relación de equivalencia ${ }^{2}$. Una relación de equivalencia es aquella que tiene la propiedad de ser 1) simétrica, 2) transitiva y 3) reflexiva. La relación de equivalencia define unívoca y exclusivamente los elementos de un universo dado. En esta forma total de intercambio se elimina el carácter arbitrario con el que estaba afectado la forma singular de valor. Si la mercancía A se encuentra en una relación de equivalencia con el resto del conjunto de las mercancías y no se reduce a tener una relación de intercambio con una mercancía singular, entonces, el valor manifestado en esta relación de equivalencia tiene que ser algo de la cosa misma, es decir, de la mercancía intercambiada, y no algo producto del $\mathrm{azar}^{3}$. El valor de una mercancía es así algo objetivo — en contra de las corrientes marginalistas más extremas-y, a su vez, algo no físico sino, más bien, social. Cuando el algo con respecto al cual se relaciona la mercancía en el intercambio es el algo denominado «equivalente universal», es decir, cuando la mercancía se vende por dinero, entonces decimos que este algo objetivo de la mercancía, su valor, es expresado por el precio. En condiciones de competencia, es decir, en condiciones en los que la relación de intercambio es de estricta equivalencia, el precio de la mercancía expresará o será manifestación del valor de la misma.

Vemos que, de forma análoga a la masa en la física clásica, el valor tampoco es una magnitud detectable directamente sino que comparece, más bien, de una forma mediata. Esta forma mediata de manifestación del valor es el "precio». En el caso de la física, tampoco la masa comparece directamente, sino que lo hace

\footnotetext{
${ }^{2}$ Cf. Heinrich, Die Wissenschaft vom Wert, Münster, Westfäliches Dampfboot, 1999, p. 199.

${ }^{3}$ Es decir, la puesta en relación denominada «intercambio» no corre a cargo de un agente o de un individuo sino que es, por así decir, la entera sociedad la que siempre ya ha llevado a cabo esta puesta en relación.
} 
dentro de un juego de relaciones o proporciones ${ }^{4}$. En este caso, la propiedad de equivalencia de esta relación estará garantizada por la identidad del cuerpo con respecto al cual se miden las fuerzas. La masa es el lugar vacío que únicamente se define dentro de un juego de relaciones con otras masas y variables empíricas. El valor es lo socialmente objetivo de una mercancía, lo sensiblemente suprasensible de la misma, lo irreductiblemente no físico. Esta objetividad social se materializa en el intercambio en el que el valor toma la forma general de valor (Allgemeine Wertform). Es decir, el trabajo privado de los productores será reconocido como trabajo socialmente necesario cuando tenga lugar la efectiva venta en el mercado a cambio del equivalente universal «dinero» 5 .

Esta es la razón de que el intercambio que tiene lugar en la forma de valor relativa (Relative Wertform), el intercambio de $x$ cantidades de la Mercancía $A$ por $y$ cantidades de la mercancía $B$, no reconozca como socialmente necesarias los trabajos de los productores de las mercancías $A$ y $B$. En el intercambio de la mercancía $A$ por la mercancía $B$ lo único que se reconoce es el trabajo del productor de $A$ por el productor de $B$ y el trabajo del productor de $B$ por el productor de $A$. Es el intercambio de la mercancía por el dinero, resultado de la forma total del valor, es decir, la venta de la misma, lo que certifica el trabajo de la mercancía vendida como socialmente necesaria. Y cuando decimos que la mercancía tiene que ser vendida entendemos que es vendida bajo condiciones de competencia. Si no fuese esto así, el trabajo reconocido no sería social sino particular: trabajo reconocido a esta u otra empresa (situaciones de oligopolio), trabajo reconocido por este u otro consumidor (situaciones de oligopsonio).

El valor es aquel componente relacional o estructural que se materializa, a través del intercambio, en el precio. Esto no significa, sin embargo, que todo el valor tenga su origen en el intercambio ${ }^{6}$. Es decir, si bien es cierto que todo

\footnotetext{
${ }^{4}$ Relaciones que pueden expresarse mediante una ecuación de segundo grado, como en la estática, o mediante una ecuación de segundo grado o superior, como en la dinámica.

${ }^{5}$ En el caso de la física, la elección de la unidad de medida (p.e. el Kg.) equivaldrá al surgimiento del equivalente universal dinero.

${ }^{6}$ De forma análoga, teníamos que el problema al que se hacía frente Kant en la Kritik der reinen Vernunft consistía en distinguir la realización del conocimiento de su origen o forma. Cf. I. Kant, Kritik der reinen Vernunft, Berlín y Leibniz, Akademie Verlag, Vol. 3, p. 27: «Wenn aber gleich alle unsere Erkentniss mit der Erfahrung anhebt, es entspringt sie darum doch nicht eben alle aus Erfahrung».
} 
valor únicamente se realiza en el intercambio (la experiencia) no por ello tiene todo él su origen en el intercambio (la experiencia). La forma del valor, aquel valor que no tiene su origen en la experiencia, aquello que está siempre ya supuesto en todo valor, es el plusvalor. Y dado que el plusvalor es algo que se explica en y desde el proceso de producción, resultará que el proceso de producción será la forma del proceso de circulación de la mercancía. En esta lectura los empiristas de Kant serán, en Marx, los Burgueses (Bourgeois). Estos consideran que dado que el plusvalor la realizan en la venta de la mercancía su origen tiene que ser también la venta ${ }^{7}$. Marx, de manera análoga a Kant, distinguirá entre la realización del plusvalor, por un lado, y su hacerse (machen) $\mathrm{u}$ originarse (entspringen) ${ }^{8}$, por el otro. Tanto Marx como Kant reconocen así la necesidad de distinguir entre la realización o materialización de una estructura y la estructura misma.

\section{Valor de uso y Valor de Cambio}

Ya hemos dicho que el valor es algo que se realiza, es decir, se reconoce como social, en el proceso de intercambio. Necesitamos hacer ahora algunas observaciones sobre lo que entendemos bajo el concepto de «valor». La lectura habitual del $D K$ tiende a distinguir entre el valor de uso (inmediato) y el valor de cambio de la mercancía. El valor de uso (la Gebrauchswert), el soporte material de la mercancía, es su lado físico. El valor de cambio (Tauschwert) de la mercancía sería aquello que hasta ahora hemos denominado valor. En la forma relativa de valor « $x$ cantidades de la mercancía $A=y$ cantidades de la mercancía $B »$, la mercancía $A$ haría uso del soporte material de la mercancía $B$ para

${ }^{7}$ Con ello no tendrán otra forma de explicar la ganancia del capitalista que recurriendo a la pérdida de algún otro agente económico. De este modo la ganancia se convierte en algo fortuito y, por esta misma razón, en algo inexplicado, en un hecho absolutamente excepcional.

${ }^{8}$ Cf. K. Marx, Das Kapital. Kritik der politischen Ökonomie. Dritter Band, Berlín, Dietz Verlag, 1987, p. 48: «Der beim Verkauf der Ware realisierte Wertüberschuß der Mehrwert erscheint dem Kapitalsiten daher als Überschuß ihres Verkaufpreises über ihren Wert, statt als Überschuß ihres Werts über ihren Kostpreis, so daß der in der Ware steckende Mehrwert sich nicht durch ihren Verkauf realisierte, sondern aus dem Verkauf selbst entspringt» (subr. nuestro). Cf. también Ibid. p. 147: «In dem Prozeß der Zirkulation vergißt er [der Bourgeois] den Produktionsprozeß. Das Realisieren des Werts der Waren —worin das Realisieren ihres Mehrwerts eingeschlossen- gilt ihm als Machen dieses Mehrwerts» (subr. nuestro). 
expresar su valor ${ }^{9}$. Este soporte material del valor es el aspecto inmediato del valor, el valor de uso de la mercancía (Gebrauchswert). El valor de uso, dado que es algo físico y no social, quedaría fuera del estudio de la economía política. Otra cosa ocurriría con el valor de cambio (Tauschwert) de la mercancía, el cual sí se manifestaría en el proceso de intercambio siendo así algo exclusivamente social. Debido a ello, el valor de cambio sí sería parte del estudio de la economía política.

Esta es la lectura habitual de la primera sección del primer tomo del $D K$ tanto de los autores marxistas como de los autores críticos con la obra de Marx ${ }^{10}$. Frente a ello comencemos señalando que en el análisis de la forma de valor de Marx no se trata de abstraer de la forma inmediata del valor sino, más bien, de mostrar aquello que siempre ya se presupone en el mercado, es decir, aquello que es el origen del intercambio. Es decir, en el análisis de Marx no se trata tanto de

${ }^{9}$ Del mismo modo que, haciendo uso de un ejemplo del propio Marx, en la relación de Peter con Paul, la materialidad de Paul le sirve a Peter como forma de manifestación del género «ser humano» y, de ahí, como forma para que Peter se relacione consigo mismo como "ser humano». Cf. K. Marx, Das Kapital. Kritik der politischen Ökonomie. Erster Band, Berlín, Dietz Verlag,1969, p. 67: «In gewisser Art geht's dem Menschen wie der Ware. Da er weder mit einem Spiegel auf die Welt kommt noch als Fichtescher Philosoph: ich bin ich, bespiegelst sich der Mensch, zuerst in einem andren Menschen. Erst duch die Beziehung auf den Menschen Paul als seinesgleichen bezieht sich der Mensch Peter auf sich selbst als Mensch. Damit gilt ihm aber auch der Paul mit Haut und Haaren, in seiner paulinischen Leiblichkeit, als Erscheinungsform des Genus Mensch».

${ }^{10}$ Una de las principales figuras críticas será el economista austríaco Böhm-Bawerk, quien, en su enfrentamiento con la teoría del valor de Marx (E. Böhm-Bawerk: «Zum Abschluß des Marschen Systems», en: F. Eberle (ed.): Marxschen Theorie 1. Zur methodischen Bedeutung des 3. Bandes des ,Kapital, Frankfurt am Main, Suhrkamp, 1973, pp. 25-129), acusará a éste de haber abstraído del valor de uso de la mercancía y no de su valor de cambio, sin que tenga más motivos para quedarse con un lado que con el otro. De este modo, según Böhm-Bawerk, toda la argumentación de Marx sería igual de acertada — es decir, igual de incompleta— si en la primera sección cambiáramos en el texto la expresión «valor de cambio» por el de «valor de uso». Es decir, si Marx fuese coherente consigo mismo tendría las mismas razones para quedarse con el Gebrauchswert y de abstraer el lado Tauschwert de la mercancía. Böhm-Bawerk llega incluso a identificar el error lógico de Marx en la confusión entre «abstraer una circunstancia en general» (Abstraktion von einem Umstande überhaupt) y «abstraer las especiales modalidades bajo las que comparece esta circunstancia" (Abstraktion von den speziellen Modalitäten, unter denen dieser Umstand auftritt). Es decir, en el caso de la Gebrauchswert, Marx «abstrae un circunstancia en general» cuando lo que debería hacer es «abstraer las especiales modalidades bajo las que comparece esta circunstancia». Con la primera modalidad de abstracción se elimina el lado de la Gebrachswert de la mercancía, con la segunda, en cambio, no. 
Wertabstraktion, es decir, de abstraer de la forma inmediata del valor, como de Wertverhältnis ${ }^{11}$, de constatar lo que en el proceso de intercambio comparece. Es cierto que Marx tiende a identificar el lado de la Gebrauchswert con las características físicas de la mercancía para, acto seguido, dado que es imposible que haya algo físicamente común entre dos mercancías intercambiadas, eliminar el lado de la Gebrauchswert de aquello común que se manifiesta en el intercambio, es decir, del valor de cambio. Pero también es cierto que este resultado obtenido por abstracción no refleja lo que tiene lugar en un proceso de intercambio mismo, es decir, en una Wertverhältnis. En él no sólo se da una igualdad en el valor de cambio de las dos mercancías ${ }^{12}$ sino que ocurre también que las dos mercancías tienen un cierto valor de uso común. El valor de uso que comparece en el intercambio no es el de esta mercancía en particular para este consumidor en particular sino, más bien, un valor de uso general. A este valor de uso no físico lo denominaremos, siguiendo a Marx, valor de uso social ${ }^{13}$.

A diferencia del valor de uso inmediato, el carácter social de la gesellschaftlicher Gebrauchswert convierte este concepto en parte de la economía políti-

${ }^{11}$ Cf. K. Marx, Das Kapital. Kritik der politischen Ökonomie. Erster Band, Berlín, Dietz Verlag, 1969, p. 65: «Sagen wir: als Werte sind die Waren bloße Gallerten menschlicher Arbeit, so reduziert unsre Analyse dieselben auf die Wertabstraktion, gibt ihnen aber keine von ihren Naturalformen verschiedne Wertform. Anders im Wertverhältnis einer Ware zur andern. Ihr Wertcharakter tritt hier hervor durch ihre eigne Beziehung zu der andern Ware» (subr. nuestro). Del mismo modo, la igualación del trabajo complejo al trabajo simple no es algo decretado por el filósofo, sino, más bien, algo que siempre ya ha ocurrido en el intercambio de una mercancia. Cf. al respecto A. Smith, An Inquiry into the Nature and Causes of the Wealth of Nations, Indianapolis, Liberty Fund, 1981, pp. 48-49: «There may be more labour in an hour's hard work than in two hours easy business; [...] But it is not easy to find any accurate measure either of hardship or ingenuity. In eschanging indeed the different productions of different sorts of labour for one another, some allowance is commonly made for both. It is adjusted, however, not by any accurate measure, but by the higgling and bargaining, of the market, according to that sort of rough equality which, though not exact, is sufficient for carrying on the business of common life» (subr. nuestro). Obsérvese la distinción entre work —algo físico—y labour —algo metafísico, es decir, no físicamente medible- en el texto de Smith.

${ }^{12}$ Se podría decir con Frege que las dos mercancías son el distinto sentido (Sinn) de la referencia (Bedeutung) «valor de cambio».

${ }^{13}$ El trabajo encargado de «crear» valor de uso social no será el trabajo de este carpintero o de este soldador sino, más bien, el trabajo de este carpintero o de este soldador en tanto que son en general trabajo útil. Cf. K. Marx, Das Kapital. Kritik der politischen Ökonomie. Erster Band, Berlín, Dietz Verlag, 1969, p. 56: «Die Arbeit, deren Nützlichkeit sich so im Gebrauchswert ihres Produkts oder darin darstellt, daß ihr Produkt ein Gebrauchswert ist, nennen wir kurzweg nützliche Arbeit». 
$\mathrm{ca}^{14}$. Y es que si bien es cierto que a la hora de constatar el intercambio de dos mercancías cualesquiera no resulta relevante el valor de uso particular de cada una, sí que comparece en el intercambio el que las mercancías tengan un valor de uso en general. Este valor de uso general es el valor de uso que tiene esa mercancía para cualquier otro consumidor en general, es decir, es el valor de uso social (gesellschaftlicher Gebrauchswert) ${ }^{15}$.

Si tanto el valor de uso social como el valor de cambio son relevantes para la economía política, surge la pregunta por la relación entre el valor de uso social y el valor de cambio. Pues bien, el valor de uso social es la condición de y proporcional al valor de cambio. Esto significa que, en primer lugar, para que algo sea intercambiado en el mercado tiene que tener un valor de uso para otro ${ }^{16}$, sólo entonces el trabajo invertido en la mercancía es trabajo socialmente necesario $^{17}$. Pero no se trata únicamente de que una mercancía no será intercambiada a menos que sea útil para alguien en general, sino que, además de ello,

${ }^{14}$ El doble carácter del valor de uso, como particular y como universal, así como la relación de este último con el valor (nosotros diríamos, con el valor de cambio) era ya expresado por Hegel en el $\$ 68$ de las Grundlinien der Philosophie des Rechts: «Die Sache im Gebrauch ist eine einzelne nach Qualität und Quantität bestimmte und in Beziehung auf ein spezifisches Bedürfnis. Aber ihre spezifische Brauchbarkeit [el valor de uso inmediato, físico o particular], sowie das spezifische Bedürfnis, dem sie dient, zugleich Bedürfnis überhaupt [el valor de uso social] und darin nach seiner Besonderheit ebenso mit anderen Bedürfnissen vergleichbar ist [a saber, en el intercambio], und darnach auch die Sache mit solchen, die für andere Bedürfnisse brauchbar sind. Diese ihre Allgemeinheit, deren einfache Bestimmtheit aus der Partikularität der Sache hervorgeht, so daß von dieser spezifischen Qualität zugleich abstrahiert wird, ist den Wert der Sache [el valor de uso social es su valor de cambio], worin ihre wahrhafte Substantialität [la objetividad social de la mercancia] bestimmt und Gegenstand des Bewußtseins ist» (G. W. F. Hegel, Grundlinien der Philosophie des Rechts, Berlín, Akademie Verlag, 1956, pp. 70-71). El análisis del valor de Ricardo considera únicamente el valor de uso inmediato y no el social. Es por ello que su análisis se limita a afirmar que el valor de uso inmediato es condición para que algo sea intercambiado y tenga así un valor de cambio en concreto. Cf. Ricardo, On the Principles of Political Economy and Taxation, Cambridge, Liberty Fund, 1951, p. 12.

${ }^{15}$ Cf. K. Marx, Das Kapital. Kritik der politischen Ökonomie. Erster Band, Berlín, Dietz Verlag, 1969, p. 55: «Um Ware zu produzieren, muss er nicht nur Gebrauchswert produzieren, sondern Gebrauchswert für andere, gesellschaftlichen Gebrauchswert».

${ }^{16} \mathrm{Y}$ no, única y exclusivamente, valor de uso inmediato, es decir, tener tales o cuales propiedades físicas, por muy abstractas que éstas sean.

${ }^{17}$ Téngase en cuenta que no estamos hablando aquí de una prioridad/posterioridad temporal. Es decir, no se trata de que primero algo tiene valor de uso social y luego es vendido en el mercado, sino que más bien, algo es vendido si y sólo si tiene valor de uso social. 
resulta que cuanto más útil resulta algo «a la sociedad» más valor de cambio tendrá. De este modo vemos que el valor de cambio es, por así decir, la manifestación o la expresión del valor de uso social ${ }^{18}$. El valor de uso social introduce así el componente de la demanda en el análisis del valor marxiano ${ }^{19}$. Esto significa que sólo una vez que hayamos pasado por el mercado, es decir, sólo una vez que hayamos comprobado lo que hemos vendido y a cuánto lo hemos vendido, sólo entonces, decíamos, podremos hablar de valor de uso social y de fuerza de trabajo socialmente necesario (invertida en tal mercancía). Esto significa, a su vez, que si bien es verdad que el valor de uso social es la condición para que tenga lugar el valor de cambio, es decir, para que se intercambie el producto ${ }^{20}$, resulta imposible hablar de valor de uso social y, con ello, de valor de cambio, sin pasar antes por el mercado. Con ello, vemos que la necesidad de que el valor sea referido al intercambio es equivalente a la afirmación del carácter social del valor de uso de la mercancía.

La teoría del valor de Marx no es una teoría sintética de la magnitud «valor» en la que partiendo de los componentes de la misma pudiera obtenerse un valor determinado del mismo, por así decir, a priori ${ }^{21}$, antes de pasar por la experiencia del intercambio. En condiciones de competencia, los valores de cambio marcados por el mercado son, por definición, valores equivalentes. El trabajo inver-

${ }^{18}$ Esta afirmación permite arrojar luz sobre la paradoja de las mercancías que, como el diamante, tienen mucho valor (de cambio) sin que aparentemente tengan apenas valor (de uso). Visto desde el valor de uso social, la paradoja llega incluso a disolverse. Dicho brevemente, si un diamante es intercambiado o es intercambiable por 100 toneladas arroz, se deberá a que socialmente es más útil que 100 toneladas de arroz.

${ }^{19}$ Evitaremos los supuestos resultados desconcertantes de esta lectura en el que se haría depender el valor de la mercancia de los caprichos de la demanda haciendo uso, al final de este mismo artículo, del mercado de capitales.

${ }^{20}$ Es decir, para que el producto sea, además de producto, mercancía.

${ }^{21}$ Cf. K. Marx, Das Kapital. Kritik der politischen Ökonomie. Zweiter Band, Berlín, Dietz Verlag, 1987, p. 381: «Weil dieser vom Arbeiter beständig reproduzierte Neuwert für ihn Quelle von Revenue bildet, bildet nicht umgekehrt seine Revenue einen Bestandteil des von ihm produzierten Neuwerts». Cf. Ibid. p. 384: «Die abgeschmackte Formel, daß die drei Revenuen, Arbeitslohn, Profit, Rente, drei «Bestandteile» des Warenwerts bilden, entspringt bei A. Smith aus der plausibleren, daß der Warenwert resolves itself, sich auflöst, in diese drei Bestandteile. Auch dies ist falsch [...]». Cf. Ibid. p. 382: «Nach diesem Quidproquo, wo die Revenue die Quelle von Warenwert wird, statt der Warenwert die Quelle von Revenue, erscheint nun der Warenwert als „zusammengesetzt» aus den verschiedenen Sorten Revenuen». 
tido en los productos intercambiados en el mercado es igual de socialmente necesario. Pero esto no significa que, en situación de competencia, a saber, cuando se intercambian mercancías equivalentes, éstas hayan de ser resultado de las mismas horas de trabajo. Y esto por dos razones. En primer lugar, porque la mercancía intercambiada puede haber sido producida por debajo o por encima del nivel medio de productividad ${ }^{22}$. En el primer caso, parte ${ }^{23}$ del trabajo invertido en el producto no será reconocido por el mercado, es decir, será socialmente no necesario. En el segundo caso se reconocerá más trabajo del que realmente está invertido en la mercancía.

La segunda razón para rechazar la lectura empirista de la ley del valor es más fundamental. El incumplimiento de esta lectura de la ley no tiene lugar únicamente para unas mercancías particulares producidas bajo ciertas condiciones de producción. El problema es más grave que la mera imposibilidad de aplicar la supuesta ley del valor en ciertos casos. El verdadero problema viene de la lectura fisicista que se hace de la ley. Según ésta, las variables de las que se afirma la ley son variables físicas, es decir, medibles con un aparato como el cronómetro. Frente a esto, ya hemos visto a raíz de la forma inmediata del valor que las propiedades físicas de las mercancías no entran dentro del estudio de la economía política. Es decir, no son las horas concretas, medibles empíricamente mediante un reloj, los que se igualan en la ley del valor. La ley del valor es una ley económica y no, tal y como pretende la versión a la que nos enfrentamos, física. La cuestión es ahora, ¿qué forma tiene la versión económica de la ley del valor?

La lectura de la ley del valor que defenderemos toma como punto de partida el hecho de que en el mercado, en condiciones de competencia, se intercambian trabajos igual de socialmente necesarios. Esto significa que el trabajo equivalentemente intercambiado ${ }^{24}$ no es el trabajo particular de la mercancía intercambiada en cada caso, sino que es, más bien, trabajo humano abstracto. Es decir, de mane-

${ }^{22}$ Suponemos que la demanda se limita a absorber los productos producidos por el nivel medio de productividad. Para la discusión de los dos casos restantes — la demanda absorbe más/menos que lo producido por el nivel medio de productividad— véase el capítulo 10 del tercer tomo del Capital.

${ }^{23}$ A saber, la parte del trabajo realizado que sobresalga del socialmente necesario.

${ }^{24}$ Cuya proporcionalidad con el valor afirma la ley del valor 
ra análoga a lo que ocurría con las restantes mercancías, tampoco podemos saber de antemano, es decir, antes de su venta en el mercado, cuál es el valor de la mercancía «fuerza de trabajo». Es más, para ver cuánto trabajo está siendo reconocido como necesario por el mercado no sólo se ha de considerar el valor de la fuerza del trabajo, es decir, el precio de esa mercancía en el mercado ${ }^{25}$, sino que hay que considerar, a su vez, el valor extra realizado por los trabajadores para el capitalista. La forma que adquiere este valor extra o plusvalor (mehrwert) una vez que ha sido materializado en el mercado es el de «beneficio» (profit $)^{26}$. Esto significa que sólo podremos saber cual es el plusvalor extraído después de que hayamos vendido la mercancía, es decir, después de que sepamos cuál es el beneficio realizado.

Lejos de ser una magnitud física, inmediatamente medible, extensa, la fuerza de trabajo socialmente necesaria reconocida en mercancía es una magnitud estructural, relacional o intensiva. No se trata únicamente de que, dependiendo de la distinta intensidad o dificultad de la tarea, dos horas de labor pueden expresar más o menos trabajo ${ }^{27}$. La cantidad de trabajo reconocido como socialmente necesario, es decir, el trabajo que formará valor ${ }^{28}$, dependerá del valor de uso social que se le reconozca a la mercancía producida por ese trabajo en el mercado y de ahí que dependa de, por así decir, los caprichos a los que obedezcan los movimientos del mercado ${ }^{29}$. La ley del valor afirma la proporcionalidad entre el

${ }^{25}$ Precio denominado «salario». Cf. K. Marx, Das Kapital. Kritik der politischen Ökonomie. Zweiter Band, Berlín, Dietz Verlag, 1987, p. 379: «[...] der Wert der Arbeitskraft —d.h. der adäquate Verkaufpreis dieser Ware».

${ }^{26}$ Dado que el mercado no nos dice directamente qué parte del beneficio total se debe a cada trabajador, no tendremos otra alternativa que hacer la suma de los salarios de todos los trabajadores y los beneficios, cuando queremos ver cuánto trabajo ha sido reconocido como necesario por el mercado.

${ }^{27}$ Hemos visto en la nota 11 que esto formaba ya parte de la interpretación fisicalista de la teoría del valor de A. Smith. Desde la teoría no fisicalista del valor, no será cierto que una trabajo más intenso implique un valor mayor pero si que un valor mayor supondrá, en situaciones estructurales o de equilibrio, una desventaja mayor. Volveremos sobre este punto cuando discutamos la tesis de la constancia de la tasa de plusvalor de Marx.

${ }^{28}$ Será wertbildende Arbeit.

${ }^{29} \mathrm{Si}$ de un dia para otro «se pone» de moda el consumo de un producto A, la misma cantidad física de trabajo invertido en esa mercancia representará más trabajo, por así decir, metafísico. Si es el trabajador el que se beneficia de esta moda, el movimiento de la fuerza de trabajo se encargará de que en este sector el valor (el salario) vuelva a expresar la desventaja (por así decir, el esfuerzo) asociado a este trabajo. Lo mismo ocurrirá, mutatis mutandis, si es el capitalista el que se beneficia de la moda. 
trabajo socialmente necesario invertido en una mercancía y su valor. Este valor, bajo el supuesto de la competencia, es expresado por su precio o, podríamos decir incluso, no es otra cosa que el precio.

La ley del valor no afirma que el trabajo sea la fuente de toda riqueza (Reichtum), sino que lo es, más bien, del valor ${ }^{30}$. El trabajo es aquello que media entre la necesidad subjetiva (subjektives Bedürfnis) y su satisfacción (Befriedigung) por el objeto ${ }^{31}$. En el caso del trabajo socialmente necesario, la necesidad será una necesidad social y su satisfacción se llevará a cabo por un objeto de cuyo análisis se ocupa el $D K$ : la mercancía. Decir que la necesidad satisfecha por la mercancía es una necesidad social no es otra cosa que afirmar que la mercancía tiene valor de uso social. Y esto último, a saber, que la mercancía tiene valor de uso social, está incluido en la afirmación «la mercancía es producto de trabajo socialmente necesario» ${ }^{32}$.

\section{Sobre el denominado problema de la transformación de los valores en precios}

Antes de pasar a ver las implicaciones que tiene nuestra lectura del primer tomo del $D K$ en el denominado problema de la transformación (Verwandlung) del tomo tercero, vamos a detenernos brevemente en un punto cuya aclaración hará que nos desviemos, en parte, de la versión marxiana del problema de la transformación. Nos referimos a la afirmación de la constancia de la tasa de explotación o plusvalor ${ }^{33}$.

\footnotetext{
${ }^{30}$ Cf. K. Marx, : Grundrissen der Kritik der politischen Ökonomie, Berlín, Dietz Verlag, 1953, p. 803.

${ }^{31}$ Cf. G. W. F. Hegel Grundlinien der Philosophie des Rechts, Berlín, Akademie Verlag, 1956, $\$ 189$, pp. 169-170.

${ }^{32}$ Algo que expresará Marx así: «die gesellschaftlich notwendige Arbeitszeit [ist] [...] die Arbeitszeit, die erheischt ist, [a] unter dem gegebnen Durchschnitt der gesellschaftlichen Produktionsbedingungen [b] das gesellschaftlich erheischte Gesamtquantum der auf dem Markt befindlichen Warenspezies zu erzeugen» (en Marx [1987a] p. 654). La primera parte de la definición (la parte [a]) hace referencia al lado de la oferta o del proceso de producción de la mercancía. La segunda parte (la parte $[\mathrm{b}]$ ), hace referencia a la demanda, es decir, al valor de uso social.

${ }_{33}$ Cf. K. Marx, Das Kapital. Kritik der politischen Ökonomie. Dritter Band, Berlín, Dietz Verlag, 1987, pp. 151-163.
} 
Marx afirma la constancia de la tasa de explotación para los distintos sectores productivos al comienzo del capítulo octavo del tercer tomo del $D K^{34}$. A la hora de demostrar su afirmación, Marx se reducirá a remitir al trabajo de A. Smith ${ }^{35}$ argumentando que en éste ya ha quedado suficientemente demostrada la verdad de tal tesis. En contra de lo que afirma Marx, lo cierto es que el texto de Smith no habla de las distintas tasas de explotación del trabajo sino que lo hace, más bien, de las diferentes ventajas (advantages) y desventajas (disadvantages) de los distintos trabajos. Es decir, el capitulo no afirma la proporcionalidad entre la plusvalía y el capital variable ${ }^{36}$ sino que lo que afirma es, más bien, la proporcionalidad entre la desventaja asociada a un trabajo y el salario pagado por él. Si tenemos en cuenta que el salario recibido por un trabajo puede considerarse como una ventaja —o desventaja — asociada a él, la afirmación de Smith se convertiría en la siguiente: en un mercado con movilidad perfecta de la mano de obra habrá una tendencia (tendency) a que se igualen las ventajas y desventajas asociadas a los trabajos ${ }^{37}$. Smith recoge en cinco puntos las desventajas, distintas al salario, que están asociadas a un trabajo ${ }^{38}$. En principio parecería que la desventaja de, por ejemplo, un riesgo asociado a un trabajo es algo subjetivo y difícil de medir en término monetarios. Pero esto no es así. En condiciones de competencia, el mercado expresa, mediante el salario, la desventaja asociada a un trabajo en concreto. En condiciones de competencia los trabajadores son indiferentes entre cambiar sus puestos y no cambiar. Esto significa que si dos trabajos se distinguen únicamente por el distinto

${ }^{34}$ Cf. K. Marx, Das Kapital. Kritik der politischen Ökonomie. Dritter Band, Berlín, Dietz Verlag, 1987, p. 151: «In diesem Kapitel wird nun voraussgesetzt, daß der Exploitationsgrad der Arbeit und daher die Rate des Mehrwerts und die Länge des Arbeitstags in allen Produktionsphären [...] von gleicher Größe [...] ist».

${ }^{35}$ En particular al capítulo décimo del primer libro del Inquiry. Cf. A. Smith, An Inquiry into the Nature and Causes of the Wealth of Nations, Indianapolis, Liberty Fund, 1981, pp. 116-159.

${ }^{36}$ Que es lo mismo que afirmar la constancia de la tasa de plusvalor. Recuérdese que .

${ }^{37}$ Cf. A. Smith An Inquiry into the Nature and Causes of the Wealth of Nations, Indianapolis, Liberty Fund, 1981, p. 116: "The whole of the advantages and disadvantages of the different employments of labour and stock must, in the same neighbourhood, be either perfectly equal or continually tending to equality».

${ }^{38}$ Estos puntos son; 1) «the agreeableness or disagreebleness of the employments themselves», 2) «the [...] expence of learning them», 3) «the constancy [...] of employment in them», 4) «the [...] trust which must be reposed in those who exercise them» y 5) «the probability or improbability of success in them». 
riesgo que está asociado a ellos ${ }^{39}$ la diferencia en el sueldo será la expresión monetaria de la desventaja «riesgo» dada por el mercado ${ }^{40}$.

El caso es que el plusvalor «creado» en el trabajo y la desventaja asociada a este mismo trabajo no tienen ninguna relación entre sí. Un trabajo puede ser más intenso que otro y producir, sin embargo, menos plusvalor. El concepto de explotación de Marx es algo económico y no, como es el caso de la desventaja de Smith, algo físico ${ }^{41}$. Esto significa que dos puestos de trabajo indiferentes entre sí pueden tener asociado un distinto plusvalor. Es decir, la masa en la que un trabajador está siendo explotado no es algo que el trabajador pueda llegar a percibir para, de este modo, introducirlo entre las variables que le permitirán decidir entre cambiar de puesto o no. En definitiva, no es la constancia de la tasa de plusvalor lo que es demostrado por A. Smith sino algo muy distinto, a saber, la tendencia a la igualdad de las desventajas asociadas a los distintos trabajos.

Vayamos ahora analizar el problema al que responde la capítulo sobre la Transformación de los «valores» en precios. Hasta el capítulo 9 del tercer tomo del $D K$, Marx había supuesto que eran los mismos trabajadores los que llevaban su producto al mercado para la venta. Esto significa que los trabajadores pretenderán que la venta de su mercancía les compense de las desventajas asociadas al trabajo por ellos realizado. Acabamos de ver cuáles eran, según A. Smith, los cinco grupos que recogían estas distintas desventajas. Podemos interpretar esta tendencia a la igualación de la desventaja asociada a cada empleo como la formulación de lo que se seguiría de la libre movilidad de la mercancía fuerza de trabajo, a saber: la expresión del valor de la mercancía «fuerza de trabajo» por su precio, es decir, por el salario. Hemos visto que, de un modo análogo, la condición de

\footnotetext{
${ }^{39}$ Riesgo del que, para que todo este puzzle pueda funcionar, todos los trabajadores $-\mathrm{y}$ no sólo el trabajador que de hecho tiene es trabajo- deben de ser conscientes. Como vemos, se vuelve a suponer aquí el «enciclopédico saber» del vendedor —y del comprador- de las mercancías —en este caso de la mercancía «de la fuerza de trabajo» - del que hablaba Marx en el primer tomo del DK. Cf. K. Marx, Das Kapital. Kritik der politischen Ökonomie. Erster Band, Berlín, Dietz Verlag, 1969, p. 50 nota 5: «In der bürgerlichen Gesellschaft herrscht die fictio juris, daß jeder Mensch als Warenkäufer eine enzyklopädische Warenkenntnis besitzt».

${ }^{40} \mathrm{Y}$ no únicamente por el trabajador que de hecho tiene ese puesto. Siendo esto así, la valoración del riesgo será algo socialmente objetivo.

${ }^{41}$ Lo mismo cabría decir — si obviamos algunas vacilaciones de Marx- del concepto de trabajo en Marx y en Smith.
} 
competencia era irrenunciable cuando se quería que los precios fuesen la expresión del valor de las mercancías. En ambos casos, la competencia era el instrumento por el que se hacía cumplir la ley del valor ${ }^{42}$. En el primer caso para el mercado de la fuerza de trabajo, en el segundo, para el mercado de las restantes mercancías.

Nos falta ahora introducir ${ }^{43}$ la condición de competencia para la variable en función al cual el capitalista toma sus decisiones. Esta variable es la tasa de ganancia o el plusvalor dividido por el capital invertido:

La condición de competencia ${ }^{44}$ en este tercer mercado, a saber, el mercado de capitales, implicará la igualdad ${ }^{45}$ de la tasa de ganancia entre las distintas empresas. Esto significará que las mercancías no serán intercambiadas por más tiempo como meras mercancías ${ }^{46}$ sino como productos del capital ${ }^{47}$. Es decir, ahora serán los capitalistas y no los propios trabajadores los que lleven el producto al mercado.

${ }^{42}$ Cf. Marzoa [1983] p. 57: «El fenómeno de «oferta y demanda» no es, pues, nada contrapuesto a la ley del valor, sino que es precisamente el mecanismo a través del cual esa ley se cumple».

${ }^{43}$ Introducción que, como se está viendo, convierte a nuestra teoría en algo más concreta, por así decir, en algo más cercana a la realidad. Y es que, la falta de afinidad de una teoría con respecto a la realidad no se debe a que la teoría sea, valga la redundancia, «demasiado teórica», sino a que es, más bien, demasiado poco teórica. Cf. I. Kant, Über den Gemeinspruch: Das mag in der Theorie richtig sein, taugt aber nicht für die Praxis, Berlín y Leipzig, Akademie Verlag, Vol. 8, p. 276 al respecto. La tarea del pensamiento es, para decirlo con Hegel, la de volver concreto lo inmediato o abstracto.

${ }^{44}$ Condición de competencia que no es otra cosa que el estado de equilibrio para ese mercado. Lo mismo podríamos decir del mercado para la mercancía «fuerza de trabajo» así como para el mercado de las mercancías denominadas «bienes y servicios».

${ }^{45}$ Para ser exactos —es decir, más concretos—- deberíamos introducir aquí, tal y como hemos hecho en el caso de la mercancía «fuerza de trabajo», los factores (des)aventajadores que hacen que a un capitalista le pueda resultar indiferente invertir en dos negocios con distinta tasa de ganancia. El factor desfavorecedor principal del capital es el riesgo que está asociado a la inversión. En condiciones de movilidad perfecta entre capitales, la distinción entre la tasa de ganancia de dos inversiones que únicamente se distinguen entre sí por el riesgo será la expresión monetaria objetiva de la (diferencia) del riesgo. Recuérdese que algo análogo tenía lugar también para la mercancía "fuerza de trabajo» y sus respectivos factores desfavorecedores.

${ }^{46}$ Es decir, que las mercancías no serán llevadas al mercado, como hasta ahora, por los mismos trabajadores. 
Si a la manifestación del valor de las mercancías en un mercado competitivo en el que son los trabajadores los que llevan el producto al mercado ${ }^{48}$ hemos denominado precio de la mercancía, a la expresión del valor de las mercancías en la que se tiene en cuenta el «mercado de capitales» ${ }^{49}$ lo denominaremos, con Marx, precio de producción. Dado que el precio de producción es resultado de un mayor trabajo teórico ${ }^{50}$, resultará que será una mejor aproximación de los precios que efectivamente tienen lugar en el mercado. Esto no significa que el precio de producción sea una mejor expresión del valor de las mercancías que el mero precio. El valor, es decir, la fuerza de trabajo socialmente reconocido en la mercancía sigue estando expresado, antes y después, por aquello que hemos denominado "mero precio» ${ }^{51}$. Una vez que hemos sentado esto, podemos formular el problema de la transformación así: se trata de ver cuales son las desviaciones es decir, las transformaciones - que sufren los meros precios si introducimos la restricción de la igualdad de la tasa de ganancia para todos los sectores en el terreno de juego.

Veamos cómo tiene lugar la transformación de los meros precios de las mercancías en los precios de producción haciendo uso, en parte, de los datos del ejemplo que proporciona el mismo Marx $^{52}$. Dado que no aceptamos la cons-

${ }^{47}$ Cf. K. Marx, Das Kapital. Kritik der politischen Ökonomie. Dritter Band, Berlín, Dietz Verlag, 1987, pp. 184-185: «Die ganze Schwierigkeit kommt dadurch hinein, daß die Waren nicht einfach als Waren ausgetauscht werden, sondern als Produkt von Kapitalen, die im Verhältnis zu ihrer Größe, oder bei gleicher Größe, gleiche Teilnahme an der Gesamtmasse des Mehrwerts beanspruchen». tales».

${ }^{48}$ Es decir, se trata de la situación en la que mantenemos apagada la variable «mercado de capi-

${ }^{49}$ Es decir, una situación en la que, tal y como ocurre en el capitalismo, es el capitalista y no los trabajadores, el que lleva el producto al mercado.

${ }^{50}$ Cf. K. Marx, Das Kapital. Kritik der politischen Ökonomie. Dritter Band, Berlín, Dietz Verlag, 1987, p. 186: «Der Austausch von Waren zu ihren Werten oder annähernd zu ihren Werten erfordert also eine viel niedrigere Stufe als der Austausch zu Produktionspreisen, wozu eine bestimmte Höhe kapitalistischer Entwicklung nötig ist».

${ }^{51}$ Cf. F. M. Marzoa, La filosofía de «El capita», Madrid, Taurus, 1983, p. 66: «la teoría de los «precios de producción» no puede en absoluto sustituir como explicación de los fenómenos a la primera teoría del valor, ya que no cumple el requisito fundamental que ésta se había impuesto a sí misma, a saber: el de encontrar aquella magnitud única, aquella «sola cosa», de la que son cantidades las diversas mercancías en cuanto valores».

52 Cf. K. Marx, Das Kapital. Kritik der politischen Ökonomie. Dritter Band, Berlín, Dietz Verlag, 1987, p. 166. 
tancia de la tasa de plusvalor, no hemos seguido la propuesta de Marx — tasa de plusvalor del $100 \%$ para todos los sectores-y, en vez de ello, hemos supuesto distintas tasas de plusvalor - 75\%, 60\%, 50\%, 85\% y 100\% ${ }^{53}$ - Debido a este cambio, el plusvalor, la tasa de ganancia, el precio de la mercancía así como el Precio de coste (Kostpreis) difieren de los valores obtenidos por Marx. Siguiendo a la lectura del concepto de valor que hemos estado defendiendo, hemos denominado «Precio de las mercancías» a lo que Marx denomina en el original «valor de las mercancías» (Wert der Waren).

La tabla con los precios de las mercancías es la siguiente.

\begin{tabular}{|c|c|c|c|c|c|c|}
\hline Capitales & $\begin{array}{c}\text { Tasa de plus- } \\
\text { valor }\end{array}$ & Plusvalor & $\begin{array}{c}\text { Tasa de } \\
\text { ganancia }\end{array}$ & $c$ utilizado & $\begin{array}{c}\text { Precio de las } \\
\text { mercancías }\end{array}$ & $\begin{array}{c}\text { Precio de } \\
\text { coste }\end{array}$ \\
\hline $\begin{array}{c}\text { I. } \\
80_{c}+20_{v}\end{array}$ & $75 \%$ & 15 & $15 \%$ & 50 & 85 & 70 \\
\hline $\begin{array}{c}\text { II. } \\
70_{c}+30_{v}\end{array}$ & $60 \%$ & 18 & $18 \%$ & 51 & 99 & 81 \\
\hline $\begin{array}{c}\text { III. } \\
60_{c}+40_{v}\end{array}$ & $50 \%$ & 20 & $20 \%$ & 51 & 111 & 91 \\
\hline $\begin{array}{c}\text { IV. } \\
85_{c}+15_{v}\end{array}$ & $85 \%$ & 12.75 & $12.75 \%$ & 40 & 67.75 & 55 \\
\hline $\begin{array}{c}\text { V. } \\
95_{c}+5\end{array}$ & $100 \%$ & 5 & $5 \%$ & 10 & 20 & 15 \\
\hline $\begin{array}{c}\text { Summa } \\
390_{c}+110_{v}\end{array}$ & - & 70.75 & - & - & - & - \\
\hline $\begin{array}{c}\text { Media } \\
78_{c}+22_{v}\end{array}$ & - & 14.15 & $14.15 \%$ & - & - & - \\
\hline
\end{tabular}

Para poder transformar estos precios en los precios de producción tenemos que calcular la tasa de ganancia media de los cinco sectores productivos. Sumando los cinco capitales como si se tratara de un solo sector y dividiendo el bene-

${ }^{53}$ Lo único que hemos presupuesto a la hora de repartir las distintas tasas de plusvalor entre los distintos sectores es que cuanto más avanzado esté un sector —es decir, cuanto menor sea su composición orgánica del capital— más se explota en él la mano de obra —es decir, es mayor su tasa de explotación-. 
ficio total por 5, obtenemos la tasa de ganancia media (Durschnittsprofit) del $14.15 \%$. Para poder obtener los precios de producción (Produktionspreise) ${ }^{54}$ tenemos que aplicar la tasa media de ganancia a los cinco sectores productivos. Para ello tendremos que aumentar en un $14.15 \%$ el precio de costo de las mercancías. La tabla que recoge estos resultados junto con las desviaciones de los precios de producción con respecto a los meros precios es la siguiente:

\begin{tabular}{|c|c|c|c|c|c|c|c|}
\hline Capital & $\begin{array}{c}\text { Tasa de } \\
\text { plusvalor }\end{array}$ & Plusvalor & $\begin{array}{c}\text { Tasa de } \\
\text { ganancia }\end{array}$ & $\begin{array}{r}\text { Precio de la } \\
\text { mercancía }\end{array}$ & $\begin{array}{c}\text { Precio de } \\
\text { coste }\end{array}$ & $\begin{array}{c}\text { Precio de } \\
\text { producción }\end{array}$ & Desviación \\
\hline $\begin{array}{c}\text { I. } \\
80_{\mathrm{c}}+20_{\mathrm{v}}\end{array}$ & $75 \%$ & 15 & $14.15 \%$ & 85 & 70 & 84.15 & -0.85 \\
\hline $\begin{array}{c}\text { II. } \\
70_{\mathrm{c}}+30_{\mathrm{v}}\end{array}$ & $60 \%$ & 18 & $14.15 \%$ & 99 & 81 & 95.15 & -4.85 \\
\hline $\begin{array}{c}\text { III. } \\
60_{\mathrm{c}}+40_{\mathrm{v}}\end{array}$ & $50 \%$ & 20 & $14.15 \%$ & 111 & 91 & 105.15 & -5.85 \\
\hline $\begin{array}{c}\text { IV. } \\
85_{\mathrm{c}}+15_{\mathrm{v}}\end{array}$ & $85 \%$ & 12.75 & $14.15 \%$ & 67.75 & 55 & 69.15 & +1.4 \\
\hline $\begin{array}{c}\mathrm{V} . \\
95_{\mathrm{c}}+5_{\mathrm{v}}\end{array}$ & $100 \%$ & 5 & $14.15 \%$ & 20 & 15 & 29.15 & +9.15 \\
\hline
\end{tabular}

La suma de las desviaciones de los precios de producción con respecto a los meros precios es igual a cero. Suponiendo un mercado de capitales competitivo, los sectores con una tasa de plusvalor mayor a la media venderán su mercancía por debajo de su valor o mero precio, mientras que los sectores con una tasa de plusvalor menor a la media lo harán por encima del valor de sus mercancías. La diferencia entre el precio de producción y mero precio es igual a la diferencia entre el plusvalor de cada sector y el beneficio del capitalista en ese sector ${ }^{55}$. Teniendo en cuenta que un sector estará más adelantado cuanto más capital cons-

${ }^{54}$ En su tabla, Marx denomina "precio de la mercancía» (Preis der Waren) a lo que, de acuerdo a la definición del propio Marx, debería haber llamado, "precio de producción» (Produktionspreis). Nosotros seguiremos la definición dada por Marx (en K. Marx, Das Kapital. Kritik der politischen Ökonomie. Dritter Band, Berlín, Dietz Verlag, 1987, p. 167 y 175) y denominaremos «precios de producción" a los precios que son resultado de aplicar una misma tasa de ganancia —a saber, la tasa de ganancia media intersectorial— a todos los sectores de producción.

${ }^{55}$ Beneficio que es el mismo en todos los sectores debido a que la tasa de ganancia y el capital invertido son iguales en todos ellos por construcción. 
tante utilice en relación a capital variable — es decir, cuanto más maquinaria y menos mano de obra emplee-, vemos que los sectores más adelantados ${ }^{56}$ son aquellos cuyas mercancías se venden en mayor proporción por encima de su mero precio $^{57}$. La suma de las desviaciones es, en cualquier caso, igual a cero y, por ello, la suma de los precios de producción es igual a la suma de los meros precios ${ }^{58}$.

Algo análogo ocurría con la tasa de productividad cuando lo que se trataba de comparar eran los distintos niveles de adelanto dentro de un mismo sector. Las empresas con mayor productividad lograban vender su producto por un precio superior a la suma de su costo más el plusvalor ${ }^{59}$ mientras que las menos productivas tenían que vender su producto a un precio menor ${ }^{60}$. La tasa de productividad permitía una comparación intrasectorial del nivel de adelanto de las distintas empresas y explicaba el hecho de que unas lograban realizar más valor que el socialmente necesario mientras que otras conseguían realizar menos.

El problema de la tasa de productividad era que no permitía una comparación intersectorial del nivel de adelanto. Esta tasa tiene en cuenta el lado físico del producto ${ }^{61}$ por lo que no puede aplicarse entre distintos sectores en los que, por definición, se producen mercancías físicamente diferentes. En vez de ello será la composición orgánica del capital la que, desde el lado del valor ${ }^{62}$, permita una

${ }^{56} \mathrm{El}$ orden, de más a menos adelantado, es el siguiente: V, IV, I, II, III.

${ }^{57}$ Haciendo uso del orden de los sectores de la nota anterior, las variaciones de los precios de producción con respecto al mero precio son: $+9.15,1.4,-0.85,-4.85$ y -5.85 .

${ }^{58}$ Algo que Marx expresa así: «die Summer der Produktionspreise des gesellschaftlichen Gesamtprodukts [ist] gleich der Summe seiner Werte» (en K. Marx, Das Kapital. Kritik der politischen Ökonomie. Dritter Band, Berlín, Dietz Verlag, 1987, p. 182). Resulta innegable por la cita que Marx está pensando en los meros precios utiliza el término Wert.

${ }^{59}$ Con ello, los capitalistas de estas empresas obtenían lo que Marx denominaba un Surplusprofit (Cf. K. Marx, Das Kapital. Kritik der politischen Ökonomie. Dritter Band, Berlín, Dietz Verlag, 1987, p. 188).

${ }^{60}$ Obviamente este estado no puede estable. Teniendo en cuenta que hemos presupuesto que una menor composición orgánica del capital implica una mayor tasa de explotación —es decir, la empresa que más maquinaria utiliza en relación a la fuerza de trabajo, más consigue explotar esta fuerza de trabajo- - las empresas que venden sobre el mero precio obtendrán una mayor tasa de beneficio. Esto atraería capital a esta empresa disminuyendo la mayor tasa de beneficio hasta que ésta se iguale a la media.

${ }^{61}$ De hecho, la tasa de productividad se define como la cantidad producida del producto por horas de trabajo invertidas en él.

${ }^{62}$ Es decir, desde el punto de vista del precio. La composición orgánica del capital se distingue 
comparación intersectorial del nivel de adelanto de los distintos sectores. Hacien-
do uso de la composición orgánica del capital será posible decir, no ya que una
empresa sea más productiva que otra, sino que un sector está más adelantado que

de la composición técnica del capital precisamente en que el segundo considera el cociente entre el capital variable y el capital constante desde el punto de vista de su materialidad —es decir, de aquello que denominábamos valor de uso inmediato y a lo que Marx denominará ahora con el término Masse - mientras que el primero considera el capital constante y el capital variable en tanto que son expresados en términos de valor —es decir, en términos de precios. Dado que Marx no está interesado en los cambios de los precios de las mercancías que no reflejen cambios en la cantidad material de las mismas, es decir, dado que Marx no está interesado en los movimientos especulativos que pueden hacer variar el precio de las mercancías - fenómenos éstos (las denominadas Wertschwankungen) que, en tanto que diacrónicos, quedan fuera del análisis sincrónico o estructural de Marx-, la composición orgánica del capital se definirá como la composición en términos de valor del capital en la medida en que ésta es determinada por la composición técnica del capital. Cf. K. Marx, Das Kapital. Kritik der politischen Ökonomie. Dritter Band, Berlín, Dietz Verlag, 1987, p. 155: «Die Wertzusammensetzung des Kapitals, insofern sie durch seine technische Zusammensetzung bestimmt wird und diese widerspiegelt, nennen wir die organische Zusammensetzung des Kapitals». Es decir, el capital variable $v$ será el índice de la masa de trabajo que pone en juego un cierto capital (Cf. K. Marx, Das Kapital. Kritik der politischen Ökonomie. Dritter Band, Berlín, Dietz Verlag, 1987, p. 154: «Das variable Kapital dient hier also [...] als Index der Masse der von einem bestimmten Gesamtkapital in Bewegung gesetzten Arbeit») del mismo modo que el capital constante $c$ será el índice de la masa de materias primas, maquinaria, etc. que pone en juego un cierto capital. Es decir, $v$ será proporcional a o expresión de la masa de fuerza de trabajo puesto en juego mientras que $c$ lo será, por así decir, de los medios de producción. Cf. al respecto K. Marx, Das Kapital. Kritik der politischen Ökonomie. Erster Band, Berlín, Dietz Verlag, 1969, p. 640: «Die Zusammensetzung des Kapitals ist in zweifachen Sinn zu fassen. Nach der Seite des Werts Nach der Seite des Werts bestimmt sie sich durch das Verhältnis, worin es sich teilt in konstantes Kapital oder Wert der Produktionsmittel und variables Kapital oder Wert der Arbeitskraft, Gesamtsumme der Arbeitslöhne. Nach der Seite des Stoffs, wie er im Produktionsprozess fungiert, teilt sich jedes Kapital in Produktionsmittel und lebendige Arbeitskraft [...] Ich nenne die erstere die Wertzusammensetzung, die zweite die technische Zusammensetzung». El hecho de que el papel de la composición técnica del capital sea absolutamente secundario en el $D K$, es decir, el hecho de que la composición técnica del capital solo aparece en el discurso de Marx para desaparecer, a saber, para decir que es su expresión en valor (la composición orgánica del capital) lo que nos interesa, apoya nuestra lectura de «el Capital» de Marx que giraba en torno al concepto del precio como expresión del valor. Las lecturas más materialistas de «el Capital» («materialistas» en el sentido de que consideran la stoffliche Seite de la mercancía como una variable relevante en la economía política) no consiguen explicar el papel nulo que tiene la composición técnica del capital en la obra de Marx. Una vez dicho esto, resultará claro que cuando Marx hace uso de la letra $v$ para expresar el capital variable no está pensando en las horas de trabajo introducidas por tal trabajador, sino en el salario que, en condiciones de competencia, es el precio que expresa el valor de la mercancía "fuerza de trabajo». Lo mismo ocurrirá, mutatis mutandis, con la variable $c$ que de ningún modo puede llegar a representar en Marx cosas tales como «litros de aceite» o «kg de salchichón». 
otro. El sector más adelantado tendrá una menor composición orgánica del capital y podrá vender su mercancía por encima de su valor. Los sectores adelantados podrán realizar más valor, se les reconocerá como socialmente necesario más valor que el individualmente invertido en sus mercancías. Lo contrario ocurrirá con los sectores atrasados ${ }^{63}$.

\section{EI valor y los precios de producción}

Ha sido habitual en la literatura ocupada en la contraposición entre el primer y el tercer tomo del $D K$ considerar que la teoría de los precios de producción del tercer tomo anula lo afirmado sobre el valor en el primer volumen ${ }^{64}$. En el primer tomo Marx afirmaría que el valor y, por ende, el precio de la mercancía es proporcional al trabajo mientras que en el tercer tomo se afirmaría, más bien, que el precio de producción sólo coincide con el valor de una manera casual ${ }^{65}$. Con ello nos encontraríamos ante un enfrentamiento entre dos posiciones incompatibles entre sí. El enfrentamiento, en la medida en que el primero de los contrincantes representa a la teoría y el segundo — el del tercer tomo- al movimiento efectivamente real ${ }^{66}$, habría que zanjarlo a favor de la realidad. Con ello

\footnotetext{
${ }^{63}$ Seguimos en lo fundamental de nuestra lectura a F. M. Marzoa, La filosofía de "El capita», Madrid, Taurus, 1983, p. 65: «En esta interpretación, el verdadero valor sería el "precio de producción", y la aparente "transferencia de valor» de unos sectores a otros no sería tal, sino simplemente la reducción del trabajo real concreto a trabajo abstracto o socialmente necesario, en la cual unos sectores saldrían perdiendo, porque, al estar más atrasados, emplean más tiempo real de trabajo que el que corresponde a una media social intersectorial, y otros saldrían ganando por la razón contraria».

${ }^{64}$ Uno de los primeros en apuntarse a esta crítica será Böhm-Bawerk. Aunque sólo sea para defender a Marx —y no tanto la obra de Marx que es la que, en verdad, nos interesa aquí- cabe decir que es falsa la afirmación de Böhm-Bawerk según la cual Marx primero escribiría el primer volumen para luego volver a repensar las cosas, obteniendo como resultado una teoría incompatible con el primer tomo que da lugar al tercer tomo. Lo históricamente cierto es que Marx había redactado el tercer volumen de «el Capital» antes de haber publicado el primer tomo. Cf. al respecto P. Samuelson, «Understanding the Marxian Notion of Exploitation: A Summary of the SoCalled Transformation Problem Between Marxian Values and Competitive Prices", Journal of Economic Literature (Nashville), n 2, Junio 1971, p. 399.

${ }^{65}$ A saber, cuando la composición orgánica del capital de la empresa en cuestión es igual a la media aritmética de la composición orgánica intersectorial. Cf. K. Marx, Das Kapital. Kritik der politischen Ökonomie. Dritter Band, Berlín, Dietz Verlag, 1987, p. 182.

${ }^{66}$ Los términos «Werttheorie» y «wirkliche Bewegung» son del propio Marx. Cf. K. Marx, Das Kapital. Kritik der politischen Ökonomie. Dritter Band, Berlín, Dietz Verlag, 1987, p. 162.
} 
quedaría anuladas las especulaciones marxianas sobre la ley del valor del primer domo de «el capital».

Lo cierto es que resulta desacertado plantear la contraposición entre el primer y el segundo tomos del $D K$ como una contraposición entre teoría y, por así decir, realidad. Tal y como hemos defendido en el punto anterior, la contraposición entre el primer y el tercer tomo del capital es la contraposición entre teoría y teoría, a saber, entre un discurso más abstracto y otro más concreto. Pero, y esto resulta aún más importante, no se trata estrictamente de dos teorías sino del desarrollo de una misma teoría. El discurso del tercer tomo es el desarrollo del discurso del primer tomo o, si se prefiere expresarlo así, el primer tomo está asumido en el tercero.

La forma en el que discurso del primer tomo es asumido en el segundo es análoga a la forma en la que el segundo axioma o ley del movimiento de Newton asume el primer axioma. En efecto, la primera ley del movimiento de Newton es la que define el concepto de cuerpo como inerte. En la segunda ley se habla de las fuerzas que hacen que «no se cumpla» la primera ley desviándose el cuerpo de su movimiento inercial «inicial». La segunda ley afirma la proporcionalidad entre movimiento así obtenido y la fuerza aplicada exteriormente sobre este cuerpo. A nadie que conozca estas dos leyes del movimiento se le ha pasado por la cabeza afirmar que la segunda ley, la que describe los movimientos reales de los cuerpos, refuta a la primera ley, mero producto teórico. Es cierto que la segunda ley describe mejor los movimientos de los objetos que la primera ley pero no por ello deja de tener estatuto de teoría, es decir, no por ello pasa a convertirse en «realidad» o descripción de algo en sí.

Tanto la primer ley como la segunda son teoría, es decir, realidad, y la primera está asumida en la segunda en el sentido de que el no cumplimiento de la primera ley es lo que permite poner de manifiesto la segunda ley. Es decir, es cuando no se cumple la primer ley, a saber, cuando nos encontramos con una trayectoria no inercial, cuando empezamos a preguntarnos por las fuerzas que han desviado el cuerpo de tal trayectoria. La o las fuerzas que buscaremos serán medidas a partir de su diferencia con respecto al movimiento inercial contrafáctico. La primera ley del movimiento se cumple sólo contrafácticamente o, dicho algo paradójicamente, se cumple no cumpliéndose. 
Podemos trazar ahora la anunciada analogía entre la primera y la segunda ley del movimiento y el primer y el segundo tomo del capital observando, para empezar, que el lugar que ocupa la primera ley lo ocupa el primer tomo mientras que la segunda ley cumple el papel del tercer tomo. La ley del valor es lo que se cumpliría $^{67}$ si no fuese por el movimiento del mercado de capitales ${ }^{68}$. La intensidad de la fuerza con el que actúa este mercado de capitales se mide precisamente por la desviación de los precios de producción con respecto a los meros precios. En el caso límite, es decir, cuando las fuerzas del mercado de capitales se anulan entre $s^{169}$ el precio de producción coincidirá con el mero precio ${ }^{70}$. De este modo, los meros precios expresan el movimiento inercial o contrafáctico de las mercancías, movimiento con respecto al cual sería posible detectar los efectos originados por los movimientos del mercado de capitales.

El problema de este modelo, problema que es más empírico que conceptual, consiste en que, en el capitalismo ${ }^{71}$, lo que de hecho se manifiestan no son los meros precios sino, más bien, los precios de producción. El esquema de Marx no sirve para calcular empíricamente los meros precios ni las desviaciones de los mismos con respecto a los precios de producción. Pero esto no significa que el trabajo teórico de Marx, trabajo del que nos hemos ocupado en esta artículo, sea por ello desechable. El medio en el que se mueve y desde el que se ha de juzgar

${ }^{67}$ Nótese el sentido contrafáctico, afín a la primera ley, de este condicional simple.

${ }^{68}$ Estos movimientos equivalen a las fuerzas de las que habla la segunda ley.

${ }^{69} \mathrm{Y}$ esto significa, en términos de Newton, cuando el efecto de las fuerzas tiene como resultado, por así decir, inercial, coincidiendo así con el movimiento inercial contrafáctico, tendremos el equilibrio propio de la estática. Esta coincidencia entre el efecto de las fuerzas y el movimiento contrafáctico inercial tiene su traducción en Marx en la coincidencia de la tasa de ganancia de la empresa de cada caso — expresión de su movimiento inercial o previo a los fuerzas del mercado de capitales - con la tasa media intersectorial — fuerzas del mercado de capitales mutuamente anuladas resultado del cálculo de la media, es decir, fuerzas cuya suma es igual a cero y se encuentran en equilibrio entre sí-.

${ }^{70}$ Cf. K. Marx, Das Kapital. Kritik der politischen Ökonomie. Dritter Band, Berlín, Dietz Verlag, 1987, p. 182: «In diesen Sphären fällt der Produktionspreis der produzierten Waren mit ihrem in Geld ausgedrückten Wert ganz oder annährend zusammen». Si tenemos en cuenta la cita reproducida en la nota 58 será inevitable concluir que Marx utiliza indistintamente las expresiones «in Geld ausgedrückten Wert» (valor expresado en dinero) y «Wert» (valor).

${ }^{71}$ Marx dirá incluso «in höhere Entwicklung der kapitalistischen Produktionsweise» (en K. Marx Das Kapital. Kritik der politischen Ökonomie. Dritter Band, Berlín, Dietz Verlag, 1987, p. 190) convirtiendo en algo histórico lo que es resultado de un análisis estrictamente estructural. 
el trabajo de Marx es conceptual y no empírico. Hemos tratado de mostrar, en sus rasgos principales, el carácter impecable de esta mediación o construcción conceptual que se esconde detrás de lo que inmediatamente se da en el capitalismo, a saber, detrás de los precios de producción ${ }^{72}$. El trabajo de Marx no es el de deducir empíricamente los precios de producción, sino el de mostrar el horizonte conceptual desde el que estos adquieren sentido. El hecho de que, en este trabajo conceptual, Marx llega a hacer uso de tablas y expresiones numéricas ha llevado a pensar a casi todos, por no decir a todos, los intérpretes del $D K$ que Marx intentaba resolver un problema técnico — el cálculo del precio de producción que derivable de la ley del valor y del movimiento del capital— por medios empíricos -mediante el cálculo de las horas de trabajo invertidas en la producción de tal mercancía, más los MW utilizados en su producción, etc.- - Creemos haber demostrado a lo largo de este artículo que esta lectura del $D K$ no se sostiene.

Con la introducción del mercado de capitales Marx propone un nuevo plano material en el que se materializa la estructura de cuya exposición se ocupa el $D K$. Para ver que, efectivamente, esta nueva materialización lo es de la misma estructura tenemos que mostrar el carácter isomorfo de las dos materializaciones. Estos dos planos ${ }^{73}$ son el mercado de mercancías y el mercado de capitales ${ }^{74}$. Pues bien, si los precios de producción son la materialización de algo a lo que llamaremos

72 Cf. K. Marx, Das Kapital. Kritik der politischen Ökonomie. Dritter Band, Berlín, Dietz Verlag, 1987, p. 208: «der Produktionspreis eine schon ganz veräußerliche und prima facie begriffslose Form des Warenwerts ist, eine Form, wie sie in der Konkurrenz erscheint, also im Bewußtsein des vulgären Kapitalisten».

${ }^{73}$ De hecho, encontramos en el capital tres planos isomorfos, el mercado de las mercancías, el de la fuerza de trabajo y el de capitales. Sin embargo, si tenemos en cuenta que la fuerza de trabajo es una mercancía más, podremos quedarnos únicamente con dos planos isomorfos y trazar así la analogía con la lingüística sin más complicaciones.

${ }^{74}$ En la lingüística los planos isomorfos son el plano del significante y el plano del significado. Las aserciones sobre la identidad/diferencia de los elementos de un plano se realizarán remitiéndonos a cambios de identidad/diferencia de los elementos del otro plano. Es decir, la diferencia/identidad fonológica de dos realizaciones materiales fonéticas se hará comprobando la identidad/diferencia "producida» en el plano del significado. Así, dos sonidos son estructuralmente distintos, es decir, son materializaciones de fonemas distintos, si el hecho de poner uno en el lugar de otro implica cambios en el significado de las palabras. Del mismo modo, dos materializaciones lexemáticas son estructuralmente distintas, a saber, materializaciones de dos lexemas distintos, si están asociados a cambios del lado del significante. 
"valor de producción» y si las tasas de ganancia lo son de la "ganancia a secas», tendremos que dos precios de producción serán realizaciones distintas de dos valores de producción si implican dos «ganancias a secas» distintas ${ }^{75}$. Del mismo modo dos tasas de ganancia serán materializaciones de dos «ganancias a secas» distintas si implican cambios en los «valores de producción». Conseguimos así delimitar segmentos de indiferencia alrededor de cada "ganancia a secas» y de cada "valor de producción» del mismo modo que, en el análisis estructural del lenguaje, hay todo un segmento de indiferencia sonora dentro del fonema $[\mathrm{a}]^{76}$. Todo esto implicará que cuando nos encontremos fuera del umbral de indiferencia en uno de los planos en el otro también nos debemos encontrar fuera del umbral ${ }^{77}$. Es decir, si hay movimiento de capitales de un sector a otro entonces en este último sector los precios no serán realización material de los valores de producción. El fenómeno así descrito, cuando el ajuste que tiende a igualar la tasa de ganancia se demora más de lo que es habitual en condiciones de competencia, es la especulación.

\section{Bibliografía}

BöHM-BAwerk, E. (1973). Zum Abschluß des Marschen Systems, in «Marxschen Theorie 1. Zur methodischen Bedeutung des 3. Bandes des `Kapital», Edición de F. Eberle, Frankfurt am Main.

BORTKEVI, V. (1976). Wertrechnung und Preisrechnung im marxschen System, Achenbach, Hegel, G. W. F. (1956). Grundlinien der Philosophie des Rechts, edición de J. Hoffmeister, Berlín. tales.

${ }^{75}$ Es decir, si con el cambio de la tasa de ganancia viene acompañado un movimiento de capi-

${ }^{76}$ Obviamente, el aumento de nivel de competitividad de los dos mercados es equivalente a la disminución de los umbrales o segmentos de indiferencia. Nótese que, debido a la relación de isomorfismo, el aumento de competitividad en un mercado implica el aumento de competitividad en el otro y viceversa.

${ }^{77} \mathrm{La}$ idea que subyace a todo este parágrafo es la de la correspondencia, expresada ya por Ricardo, entre el denominado precio natural de la mercancía y la igualdad de la tasas de ganancia a la tasa media intersectorial. Cf. D. Ricardo, On the Principles of Political Economy and Taxation, Cambridge, Liberty Fund, 1951, p. 90: «Let us suppose that all commodities are at their natural price, and consequently that the profits of capital in all employments are exactly at the same rate». Nótese que el precio natural de Ricardo es, en nuestro texto, el precio de producción. Nótese además, que la relación de implicación de Ricardo es, en nuestro caso, una relación de co-implicación. 
Heinrich, M. (1999). Die Wissenschaft vom Wert, Münster.

Herivel, J. (1965). The Background to Newton's Principia, Oxford.

KANT, I. (Ak. VIII). Über den Gemeinspruch: Das mag in der Theorie richtig sein, taugt aber nicht fir die Praxis.

MARX, K. (1953). Grundrissen der Kritik der politischen Ökonomie, Berlín.

- (1969). Das Kapital. Kritik der politischen Ökonomie. Erster Band, Vol. 23 de las «Karl Marx — Friedrich Engels Werke», Berlín.

- (1974). Zur Kritik der politischen Ökonomie, Vol. 13 de las «Karl Marx — Friedrich Engels Werke», Berlín.

- (1987). Das Kapital. Kritik der politischen Ökonomie. Zweiter Band, Vol. 24 de las «Karl Marx- Friedrich Engels Werke», Berlín.

- (1987a). Das Kapital. Kritik der politischen Ökonomie. Dritter Band, Vol. 25 de las «Karl Marx — Friedrich Engels Werke», Berlín.

MarzoA, F. M. (1983). La filosofia de "El capita», Madrid.

MeeK, L. R. (1956). Some Notes on the "Transformation Problem», in "The Economic Journal», Vol. 66, No. 261, pp. 94-107, March.

Morishima, M. y Catephores G. (1975). Is There an "Historical Transformation Problem»?, in «The Economic Journal», Vol. 85, No. 338, pp. 309-328, Jun.

Ricardo, D. (1951). On the Principles of Political Economy and Taxation, Vol. 1 de "The Works and Correspondence of David Ricardo», edición de Piero Sraffa, Cambridge.

SAMUelson, P. (1971). Understanding the Marxian Notion of Exploitation: A Summary of the So-Called Transformation Problem Between Marxian Values and Competitive Prices, Journal of Economic Literature, Vol. 9, No. 2, pp. 339-431.

SETON, F. (1957). The «Transformation Problem», in «The Review of Economic Studies», Vol. 24, No. 3, pp. 149-160, Jun.

Smith, A. (1981). An Inquiry into the Nature and Causes of the Wealth of Nations, Vol. 1 de la "Glasgow edition of the works and correspondence of Adam Smith», Edición de R.H. Campbell, A.S. Skinner y W. B. Todd, Indianapolis.

SWEEZY, P.M. (1942). The theory of capitalist development, Oxford.

Recibido: 15/03/2010

Aceptado: 4/05/2011 
